\title{
IMPLICACIONES DEL RELIEVE SOBRE LA BAJA ATMÓSFERA EN UNA ZONA TROPICAL Y COSTERA EN MÉXICO
}

\author{
Adalberto TEJEDA-MARTÍNEZ1', Carlo A. DOMÍNGUEZ-EUSEBIO², \\ Martha PÉREZ-MÉNDEZ ${ }^{1}$, Antonio LUNA DÍAZ-PEÓN ${ }^{1}$ \\ ${ }^{1}$ Grupo de Climatología Aplicada. \\ ${ }^{2}$ Instituto de Biotecnología y Ecología Aplicada Universidad Veracruzana, Xalapa, Veracruz, México. \\ atejeda.martines@gmail.com, cardomingueze@gmail.com
}

\section{RESUMEN}

Un círculo de $200 \mathrm{~km}$ de radio con centro en la nucleoeléctrica de Laguna Verde, México $\left(19^{\circ} 43,6^{\prime} \mathrm{N}, 96^{\circ} 24^{\prime} \mathrm{W}\right)$ se puede dividir en un semicírculo sobre en el Golfo de México y otro sobre el continente. Este último se compone de dos planicies costeras con lomerío: una al sur-sudoeste y otra al norte. Hacia el poniente, el terreno es escarpado en los primeros $100 \mathrm{~km}$ de distancia y plano de gran altitud (más de 2000 m) los restantes $100 \mathrm{~km}$. Los contrastes entre mar y continente, y entre los distintos tipos de relieve, generan comportamientos diferenciados en la atmósfera baja, que se exploran con datos de una campaña de mediciones realizada en el invierno de $2011 \mathrm{y}$ otra en el verano de 2015, que constaron de sondeos con sodar, con dron y globo cautivo, así como estaciones termohigrométricas de superficie. Se mostrarán ejemplos de cómo el relieve tiene efectos diferenciados en las tendencias climáticas decadales, en el comportamiento de fenómenos meteorológicos de invierno como los frentes fríos, y en un día de verano bajo el influjo de los vientos alisios no perturbados.

Palabras clave: Efectos del relieve, tendencias decadales, frentes fríos y alisios suaves.

\begin{abstract}
A circle of $200 \mathrm{~km}$ radius centered in nuclear power plant of Laguna Verde, Mexico $\left(19^{\circ} 43.6^{\prime} \mathrm{N}, 96^{\circ} 24^{\prime} \mathrm{W}\right)$ can be divided in a semicircle on the Gulf of Mexico and another on the continent. The latter consists of two coastal plains with low hills: one south-southwest and other north. To the west the terrain is steep in the first $100 \mathrm{~km}$ away and plane at high altitude $(2000 \mathrm{~m})$ the remaining $100 \mathrm{~km}$. The contrasts between sea and continent, and above this between different types of relief, generate different behaviors in the lower atmosphere, which are explored using data from a measurement campaign conducted in the winter of 2011 and again in the summer of 2015. Both them consisted of temperature and humidity soundings with sodar, drone, and tethersonde, and surface stations. This paper shows examples of how the relief has different effects on decadal climate trends, on the winter weather events such as cold fronts, and on a summer day under the soft trade winds.
\end{abstract}

Key words: Effects of relief, decadal trends, cold fronts and soft trade winds. 


\section{INTRODUCCIÓN}

Los efectos de la orografía en las condiciones atmosféricas sorprendieran a los primeros europeos que llegaron a América, pues esperaban que por su mayor cercanía al Ecuador el "nuevo mundo" sería tan tórrido que imposibilitaría la vida humana, la agricultura o la ganadería tal como eran conocidas en Europa. Al ver que las condiciones orográficas atemperan el clima, los europeos vislumbraron la posibilidad de vivir y producir de manera similar a como lo hacían en sus lugares de origen. De modo que si bien las generalidades de los efectos del relieve sobre la atmósfera son conocidos de largo tiempo atrás, no lo son tanto para una región en particular, ni tampoco sobre las tendencias climáticas, y sobre la interacción de la baja atmósfera con los sistemas sinópticos de verano y de invierno.

La región de estudio rodea el punto donde el Eje Neovolcánico alcanza la costa del Golfo de México en donde se ubica la nucleoeléctrica de Laguna Verde $\left(19^{\circ}\right.$ 43,6’N, 96² $\left.24^{\prime} \mathrm{W}\right)$, lugar cercano al desembarco en 1519 del conquistador Hernán Cortés. Un círculo de $200 \mathrm{~km}$ de radio con centro en este punto, comprende al este un semicírculo sobre el Golfo de México y otro al oeste sobre el continente. Éste último se compone de dos planicies costeras con lomerío: una al sur-sudoeste y otra al norte. Hacia el poniente, el terreno es escarpado en los primeros $100 \mathrm{~km}$ y plano de gran altitud (más de $2000 \mathrm{~m}$ ) los restantes $100 \mathrm{~km}$. Esta orografía particular es producto de dos cadenas montañosas que convergen en esta región. El límite entre la zona escarpada y el altiplano mexicano lo establecen dos de las más grandes montañas de México, el Pico de Orizaba (5600 m de altitud) y el Cofre de Perote $(4200 \mathrm{~m})$, ubicados a $100 \mathrm{~km}$ y $80 \mathrm{~km}$, respectivamente, de la costa del Golfo de México bordeando el paralelo $19^{\circ} \mathrm{N}$. En apenas $100 \mathrm{~km}$ en la horizontal se puede pasar del nivel del mar a los $5600 \mathrm{~m}$ de altitud: estamos ante una de las pendientes altitudinales más pronunciadas del mundo (Figura 1).

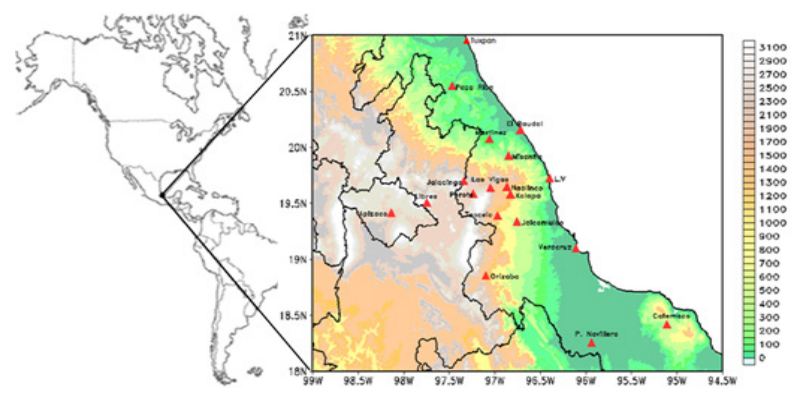

Figura 1. Región de estudio alrededor de LV (Laguna Verde). El recuadro tiene de lado $200 \mathrm{~km}$. Se señalan las estaciones climatológicas utilizadas para evaluar tendencias térmicas y de precipitación. La escala de colores indica la altitud en metros.

En la planicie costera sur la superficie es fundamentalmente agrícola (caña de azúcar, piña y otros cultivos de temporal) y pastizal para ganado vacuno (alrededor del $12 \%$ de la superficie), con reductos de selva tropical que no llegan al $5 \%$ de la superficie total. En esta zona se ubican 6 áreas urbanas de más de 50 mil habitantes e incluso una con más de 700 mil (Veracruz-Boca del Río-Medellín). Las característi- 
cas de la zona norte son ligeramente diferentes, la cubierta del suelo agrícola es principalmente destinada a caña de azúcar, cítricos, maíz, cultivos de temporal, y pastizal para ganado vacuno (menos del 10\%), mientras que la selva tropical es menor al 3\% (Alan-Ellis y Martínez-Bello, 2010). En esta zona se ubican seis áreas urbanas de más de 50 mil habitantes y la central nucleoeléctrica de Laguna Verde, que proporciona el $7 \%$ de la energía eléctrica del país. En la media montaña el bosque mesófilo abarca el $11 \%$ de la cobertura de suelo, un $17 \%$ es bosque perturbado, $17 \%$ vegetación secundaria, $37 \%$ pastizales y $18 \%$ está cubierto por asentamientos humanos con ocho áreas urbanas de más de 50 mil habitantes, incluida el área metropolitana de Xalapa con cerca de 500 mil habitantes (Williams-Linera, 2012). Entre el Pico de Orizaba y el Cofre de Perote se ubica un puerto de entrada al altiplano que ronda los 2,400 m de altitud. Una vez traspuesto éste, la porción del altiplano mexicano que comprende la zona de estudio está cubierta principalmente de bosque de coníferas y áreas semidesérticas, y dos áreas urbanas con más de cincuenta mil habitantes (Tejeda-Martínez et al., 2004). En total, la zona de estudio alberga una población de 6,1 millones de habitantes, que es el $5,4 \%$ de la población nacional.

La climatología de la región de estudio se puede resumir en la Tabla 1. Resulta de la interacción de la orografía con fenómenos meteorológicos de latitudes bajas en el semestre centrado en el verano (vientos alisios, a veces perturbados como ondas del este y ocasionalmente como tormentas tropicales), y con otros de latitudes medias en el semestre centrado en el invierno (oleadas de aire frío); en ausencia de sistemas de circulación de escala mayor, la alternancia de las brisas marina/terral domina el ciclo diurno.

La circulación sinóptica se ve muy influenciada por la orografía; por ejemplo, en el semestre centrado en el verano los vientos alisios encuentran la pared montañosa, ascienden de manera forzada para precipitar más de $1000 \mathrm{~mm}$ a barlovento $(1070 \mathrm{~mm}$ en Laguna Verde en el semestre mayo-octubre) y menos de $400 \mathrm{~mm}$ a sotavento.

Por su parte, las variaciones estacionales de la insolación en la parte de cara a la costa no dependen tanto de la declinación solar como de la nubosidad. El mes más soleado es mayo coincidiendo con el primer paso del Sol por el cenit de la zona (340 $\mathrm{W} / \mathrm{m}^{2}$ de irradiación solar global), $50 \mathrm{~W} / \mathrm{m}^{2}$ más que en el periodo lluvioso (junio a septiembre, cuando ocurre el segundo paso del Sol por el cenit) y $100 \mathrm{~W} / \mathrm{m}^{2}$ más que en invierno (octubre a febrero). En la costa, el resultado es un clima húmedo-tropical con la influencia oceánica, es decir, con lluvias nocturnas en un $80 \%$ de la precipitación anual (entre 9 P.M. y 9 A.M.).

La brisa del mar, en el verano sobrepuesta a los vientos alisios, adquiere una componente sur o sureste. Los vientos más intensos y frecuentes en Laguna Verde son los provocados por los nortes -invasiones de aire polar en el semestre centrado en el invierno-, que por la configuración del terreno adquieren una componente del oeste o del noroeste. Se presentan alrededor de cincuenta nortes al año. Los cuales provocan un descenso de la temperatura de entre 2 y $5^{\circ} \mathrm{C}$ en la costa. El Puerto de Veracruz, a $100 \mathrm{~km}$ al sur de Laguna Verde, cierra la entrada de embarcaciones de gran calaje en promedio 7 días al año (cuando los vientos rebasan los $105 \mathrm{~km} / \mathrm{h}$ ), pero los botes pesqueros no salen de la costa aproximadamente 70 días al año. 
En suma, por los sistemas de circulación que la afectan en distintas épocas del año, y por la orografía variada y su cercanía al mar, la zona es una laboratorio natural para explorar el acoplamiento entre la capa atmosférica superficial, la capa de mezcla, la capa nubosa y la circulación sinóptica, mediante el uso de información ya existente, de campañas de mediciones ex profeso y de simulaciones computacionales con modelos de mesoescala. Para más detalles ver Tejeda et al. (1988), Tejeda-Martínez et al. (2004) y Ruiz-Barradas et al. (2010).

Los contrastes entre mar y continente y, sobre éste, entre los distintos tipos de relieve, generan comportamientos diferenciados en la atmósfera baja, que se explorarán con datos de una campaña de mediciones realizada en el invierno de 2011 y otra en el verano de 2015. Estas campañas constaron de sondeos con globo cautivo y con dron, radiosondeos con globos cautivos y estaciones termohigrométricas de superficie. Los resultados de esas mediciones se usarán en este trabajo para ilustrar los efectos del relieve en una invasión de aire polar en forma de "Norte" (invierno) y en una situación típica de verano de influjo de los vientos alisios.

\begin{tabular}{|c|c|c|c|c|}
\hline Región & $\begin{array}{c}\text { Área aprox. } \\
\left(\text { miles de } \mathrm{km}^{2}\right)\end{array}$ & $\begin{array}{c}\text { Temp. máxima } \\
\text { media }\left({ }^{\circ} \mathrm{C}\right)\end{array}$ & $\begin{array}{c}\text { Temp.mínima } \\
\text { media }\left({ }^{\circ} \mathrm{C}\right)\end{array}$ & $\begin{array}{c}\text { Precipitación } \\
\text { media }(\mathrm{mm})\end{array}$ \\
\hline Llanura costera norte & 11,0 & 28,6 & 19,5 & 1575 \\
\hline Llanura costera sur & 28,5 & 29,5 & 20,3 & 1669 \\
\hline Montañosa central & 11,0 & 23,7 & 12,2 & 1575 \\
\hline Altiplano & 12,5 & 22,4 & 15,2 & 620 \\
\hline
\end{tabular}

Tabla 1. Características climáticas anuales de la zona de estudio.

\section{TENDENCIAS RECIENTES}

Desde luego, el bosquejo climático presentado en los párrafos anteriores no es estático. Hay tendencias y variaciones originadas por la variabilidad climática natural y el cambio climático antropogénico inducido a escala global, además del propio debido a alteraciones regionales en el uso del suelo. Se puede obtener una visión simplificada si se estiman las tendencias lineales de algunos índices pluviométricos y térmicos. Con este propósito se analizaron datos diarios de temperaturas máxima y mínima y precipitación de dieciocho estaciones, proporcionados por la Comisión Nacional del Agua (CNA, México) para el periodo 1980-2008 (Figura 1), de las estaciones de la Figura 1 agrupadas en planicie costera sur (LV, Veracruz, Novillero y Catemaco); planicie costera norte (Tuxpan, Poza Rica, El Raudal, Martínez y Misantla); región montañosa central (Xalapa, Naolinco, Teocelo, Jalacingo, Jalcomulco y Orizaba), y altiplano (Perote, Libres y Apizaco). Dos estaciones contaron con el 100\% de los datos y una con el 75\%; el resto presentaron una cantidad de información intermedia entre esos valores.

Los datos fueron sometidos a las pruebas de control de calidad propuestas por el ETCCDI (Grupo de Expertos en Detección e Índices de Cambio Climático). Para el cálculo de los índices climáticos se utilizó el software RClimDex. Los “outliers”, fueron corregidos o desechados mediante la consulta de boletines meteorológicos emitidos por el Servicio Meteorológico Nacional. Se utilizaron cinco de los 57 índices 
de cambio climático propuestos por la Unidad de Investigación Climática de la Universidad de East Anglia (www.cru.uea.ac.uk) y evaluados con el software StarDex: el de precipitación máxima en tres días (Tabla 2a) y los de tendencias de noches con temperatura mínima menor a $0^{\circ} \mathrm{C}$, de rango diurno de la temperatura, frecuencia de la temperatura mínima menor al percetil 10 y el de frecuencia de la temperatura máxima superior al percetil 90 (Tabla 2b). Además se agregaron dos índices relacionados con la precipitación: el índice de Gini que indica la concentración de la precipitación a lo largo del año, y que fue traspuesto de la Economía a estudios climáticos por Martín-Vide (2003), y el de la intensidad de la canícula o sequía de medio verano que se debe a Mosiño \& García (1966).

Las tendencias de los índices de precipitación se señalan en la Tabla 2(a) y de los índices térmicos en la Tabla 2(b). Todas tienen un nivel de significancia de 0,05 o menor, es decir, un nivel de confianza del $95 \%$ o superior. La precipitación máxima en tres días tiende a ser más intensa en la zona montañosa y en el altiplano que en las planicies costeras, en correspondencia con las tendencias a la intensificación de la sequía de medio verano en comparación con la parte central de la montaña o con el altiplano. El índice de Gini entre más cercano a la unidad significa una mayor irregularidad o concentración de la precipitación diaria a lo largo del año, y si se aproxima a cero significa que la precipitación se distribuye de manera casi uniforme. Así pues, tendencias negativas implican que la precipitación se va haciendo más uniforme (como en el altiplano), y positivos, más concentrada en el tiempo (planicies altas y parte media de la montaña).

Las tendencias térmicas (Tabla 2a) son congruentes entre sí y con la propensión mundial al calentamiento reportada por Panel Intergubernamental de Cambio Climático (IPCC) en su quinto informe de evaluación (http://www.ipcc.ch/pdf/assessment-report/ar5/wg1/WG1AR5_SPM_FINAL.pdf): a disminuir la frecuencia de temperaturas bajas y a aumentar la $\overline{d e}$ temperaturas altas.

\begin{tabular}{|c|c|c|c|c|}
\hline Región & $\begin{array}{c}\text { Altitud } \\
\text { media }(\mathrm{m})\end{array}$ & $\begin{array}{c}\text { Tendencia de la } \\
\text { precipitación máxima en } \\
\text { tres días (mm/década) }\end{array}$ & $\begin{array}{c}\text { Ídem índice de } \\
\text { Gini } \\
\text { (\%/década) }\end{array}$ & $\begin{array}{c}\text { Ídem índice de } \\
\text { la canícula } \\
\text { (\%/década) }\end{array}$ \\
\hline P. costera sur & 93 & 3,7 & 0,002 & 2,1 \\
\hline P. costera norte & 179 & 2,4 & 0,010 & 3,4 \\
\hline Montañosa central & 1450 & 9,0 & 0,004 & 1,1 \\
\hline Altiplano & 2411 & 8,9 & $-0,010$ & 0,1 \\
\hline
\end{tabular}

Tabla 2 (a). Para cada región (primera columna) se presentan la altitud media de las estaciones mostradas en la Figura 2 (segunda columna) y las tendencias para algunos indices de precipitación.

Se notan ligeras diferencias: las noches con heladas (temperatura menor a $0^{\circ} \mathrm{C}$ ) están disminuyendo más en la parte montañosa que en el altiplano; el rango u oscilación de la temperatura diurna se está incrementando más en las partes altas que en las planicies, al igual que la frecuencia de temperaturas altas (superiores al percentil 90), y ocurre lo contrario con las temperaturas bajas (menores al percentil 10). No obstante, no es fácil establecer en qué medida las tendencias se deben al cambio climático global y en qué otra a las modificaciones del uso del suelo. 


\begin{tabular}{|c|c|c|c|c|}
\hline Región & $\begin{array}{c}\text { Tendencia de } \\
\text { noches con } \\
\text { temperatura } \\
\text { mínima menor a } \\
0^{\circ} \mathrm{C}\end{array}$ & $\begin{array}{c}\text { Ídem de rango } \\
\text { diurno de la } \\
\text { temperatura } \\
\left(0^{\circ} \mathrm{C} / \text { década }\right)\end{array}$ & $\begin{array}{c}\text { Ídem de frecuencia } \\
\text { de la temperatura } \\
\text { mínima menor al } \\
\text { percetil } 10 \\
(\% / \text { década })\end{array}$ & $\begin{array}{c}\text { Ídem de frecuencia } \\
\text { de la temperatura } \\
\text { máxima superior al } \\
\text { percetil } 90 \\
(\% / \text { década })\end{array}$ \\
\hline P. costera sur & No aplica & 0,50 & $-0,02$ & 0,05 \\
\hline P. costera norte & No aplica & 0,11 & $-0,02$ & 0,04 \\
\hline Montaña & $-1,20$ & 0,63 & $-0,06$ & 0,04 \\
\hline Altiplano & $-0,74$ & 0,62 & $-0,03$ & 0,03 \\
\hline
\end{tabular}

Tabla 2 (b). Para cada región se presentan las tendencias térmicas.

Sesenta días al año, aproximadamente, la zona es invadida por aire polar humedecido a su paso por el Golfo de México. Los efectos diferenciados entre distintas regiones se ilustrarán con las marchas diarias de la temperara y la humedad específica y los perfiles de viento en la atmósfera baja, para los días 5 a 7 de diciembre de 2011. Se dispuso de un sodar para sondeos en la costa (Laguna Verde, LV en figura 1) y sondeos con globo cautivo en Xalapa, a $1350 \mathrm{~m}$ de altitud (Figuras 2, 3 y 4).
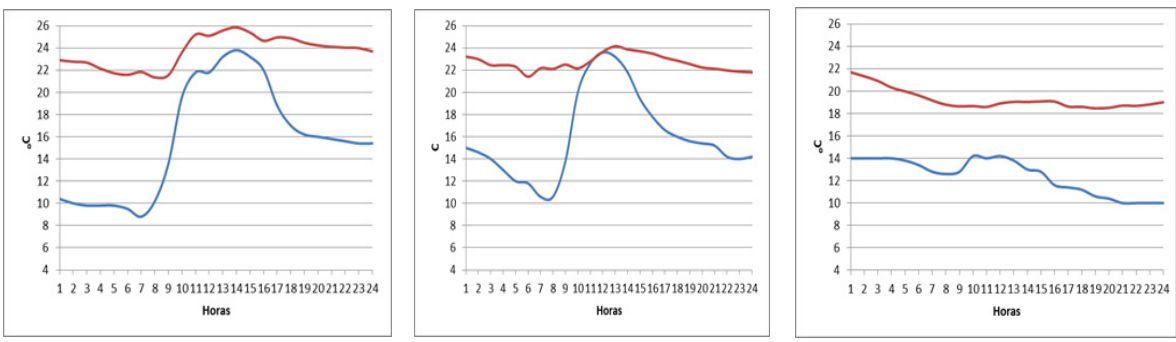

Figura 2. Marcha diaria de la temperatura $\left({ }^{\circ} \mathrm{C}\right)$ en la costa (roja, LV) y a media montaña (azul, Xalapa), a) dos días antes de la llegada del frente frío (izquierda, 5/dic/2011,), b) un día antes (centro, 6/dic/2011), y c) durante el frente (derecha, 7/dic/2011).

Dos días antes de la llegada de un frente frío la temperatura es mayor en Laguna Verde por la diferencia de altitudes; al día siguiente las temperaturas máximas se empatan porque el frente es precedido de vientos cálidos del sur (fenómeno localmente conocido como surada), pero las noches suelen ser despejadas de modo que el escape de radiación solar es mayor en la montaña, donde además no se tiene el efecto humidificador de la brisa marina, lo que explica ese contraste térmico mayor en la montaña que en la costa. La llegada del frente frío implica tanto para la montaña como para la costa, descensos casi lineales de la temperatura (ver Figura 2). La huella de la surada se aprecia en las Figuras $3 b$ y 4b, y el cambio a la llegada de la masa fría en las Figuras 3c y 4c.

\section{UN DÍA ESTABLE DE VERANO}

Del 28 de junio al 3 de julio del 2015 se realizaron sondeos mediante sensores acoplados y programados en una tarjeta (ordenador de placa reducida) Raspberry Pi, y elevados mediante dron. Se mostrará los sondeos costeros del 29 de junio. La circulación generada por la brisa terral permite el desarrollo de nubosidad frente a las costas 
del Golfo de México para las primeras horas del día. Probablemente esta nubosidad se pueda explicar por la presencia de una vaguada semi-permanente sobre el suroeste y oeste del Golfo de México para estas mismas horas durante el verano (datos de reanálisis del Global Forecast System, GFS ${ }^{1}$ ).
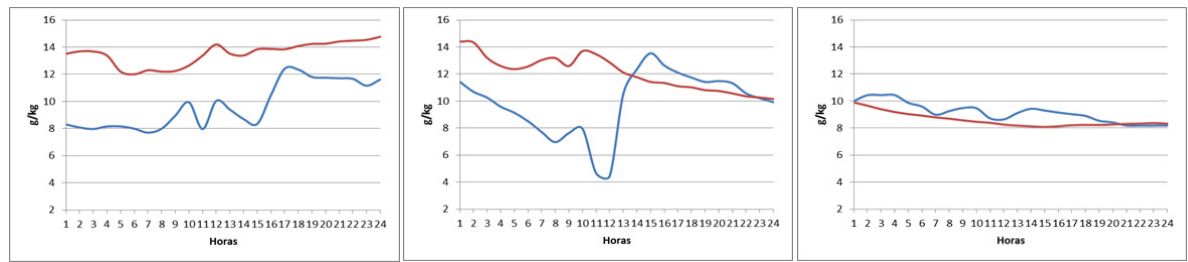

Figura 3. Marcha diaria de la humedad especifica $(\mathrm{g} / \mathrm{kg}$ ) en la costa (roja) y a media montaña (azul), a) dos días antes de la llegada del frente frío (izquierda, 5/dic/2011), b) un día antes (centro, 6/dic/2011), y c) durante el frente (derecha, 7/dic/2011)

Hacia las horas de la tarde el viento cargado de humedad desde el Golfo de México ocasiona aumento de nublados sobre la sierra. La continua entrada de humedad permite que los procesos convectivos sigan ocurriendo y da lugar a condiciones de mayor humedad en la región de estudio (Figura 5). En los primeros metros de la barrera montañosa los vientos empiezan a cambiar su dirección hacia el este y lo que ayuda al retroceso de la nubosidad hacia la zona costera durante la noche.

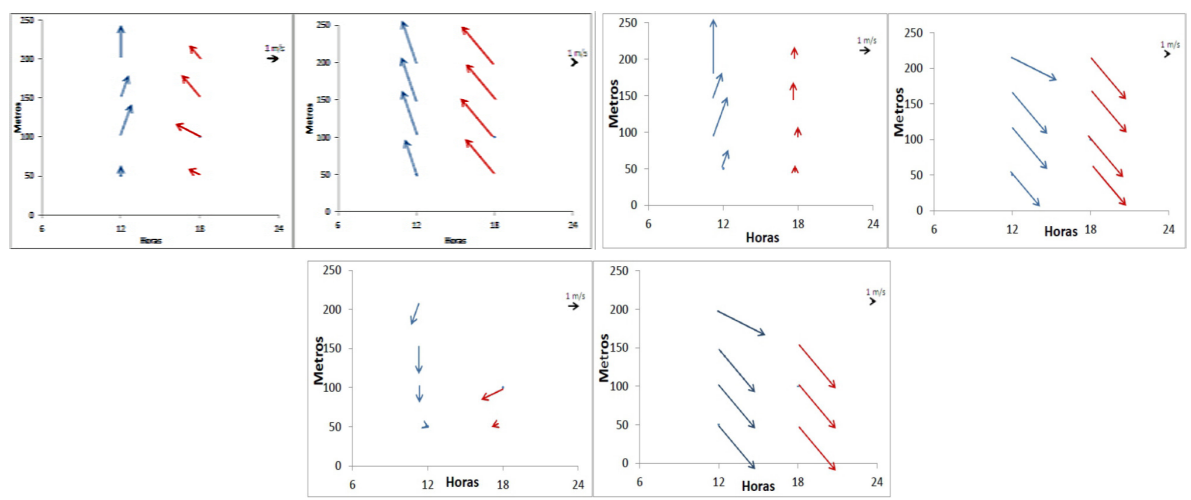

Figura 4. Perfiles verticales del viento horizontal para Xalapa (izquierda en cada recuadro) y Laguna Verde (derecha en cada recuadro) a las 12 (azul) y 18 horas locales (rojo), a) durante dos días antes de la llegada del frente frío (5/dic/2011, arriba a la izquierda), b) un dia antes (arriba a la derecha, 6/dic/2011), y c) durante el frente (abajo a la izquierda, 7/dic/2011); en Xalapa a las 18 horas no fue posible el sondeo con globo cautivo hasta 2050 $m$ de altura por la intensidad de los vientos.

Como se observa en la Figura 6, el calentamiento diurno permite que las temperaturas superficiales se eleven durante el día. Para la tarde-noche la entrada de radiación cesa y con ello la disminución de las temperaturas (este mismo patrón se observa el resto de

1 https://earth.nullschool.net/\#2015/06/29/0900Z/wind/surface/level/orthographic=-96.37,19.16,3000 
los días de esta campaña de mediciones). Los sitios de los sondeos con dron se presentan en la Figura 6c. Las condiciones de cielo despejado durante la madrugada ocasionaron que sobre la costa, (Figura 7, Flores Magón) se presentara una inversión térmica en los primeros 100 metros de la atmósfera. En niveles superiores se observó una atmósfera inestable. Al interior de la planicie costera (Santa Elena) la atmosfera presentó, entre 0-100 metros y 400-600 metros, condiciones de débil estabilidad. En el resto de la columna atmosférica las condiciones fueron neutrales. En el sitio CRPM, los primeros 600 metros de la atmosfera presentaron condiciones de inestabilidad. Para las $19 \mathrm{HL}$ en los tres sitios se observaron condiciones de inestabilidad en los primeros 600 metros.
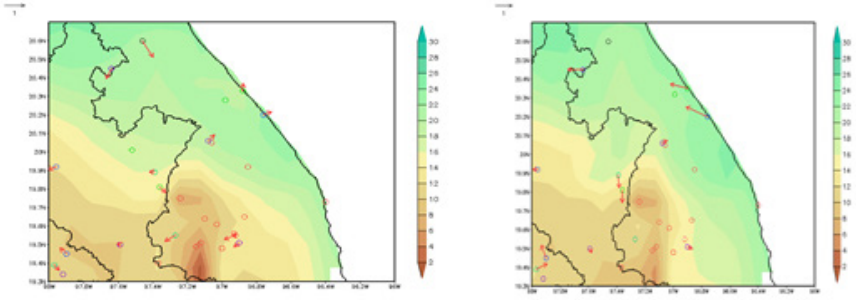

Figura 5. Humedad absoluta $\left(\mathrm{gr} / \mathrm{m}^{3}\right)$ y viento $(\mathrm{m} / \mathrm{s})$ para a) $10 \mathrm{HL}$, b) $19 \mathrm{HL}$ el 29 de junio de 2015.
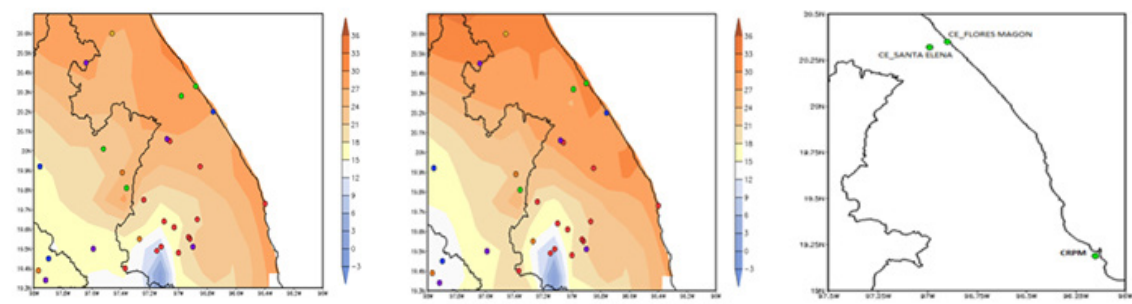

Figura 6. Temperatura $\left({ }^{\circ} \mathrm{C}\right)$ para las a) $10 \mathrm{HL}$; b) $13 \mathrm{HL}$ del 29 de junio de 2015., y c) ubicación (planicie costera norte) de los sitios donde se realizaron sondeos con dron. Entre Flores Magón y CRPM hay una distancia en línea recta de $100 \mathrm{~km}$ y entre Flores Magón y

Santa Elena, $10 \mathrm{~km}$. La curva de nivel que se muestra corresponde a la cota de $200 \mathrm{~m}$.
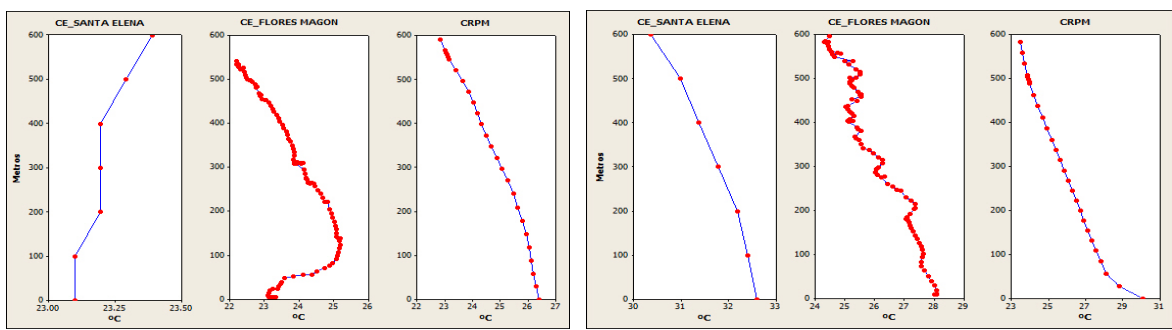

Figura 7. Perfiles de temperatura a) 07 HL y b) 19 HL 29 de junio del 2015.

El perfil de humedad absoluta $\left(\mathrm{g} / \mathrm{m}^{3}\right)$ para las 07 HL (Figura 8) en Flores Magón, permite observar cómo la capa de mezcla se extiende desde 50 a $300 \mathrm{~m}$ de altura, muy 
poco profunda debido al débil calentamiento para esta hora. Para el sitio CRPM una ligera capa de mezcla se observa de 50 a 350 m. En Santa Elena no se observan cambios significativos de la humedad con la altura. A las 19 HL es muy claro cómo la capa de mezcla se extiende desde 0 a 450 m en Flores Magón. Para el resto de los sitios la humedad disminuye con la altura en toda la columna atmosférica.
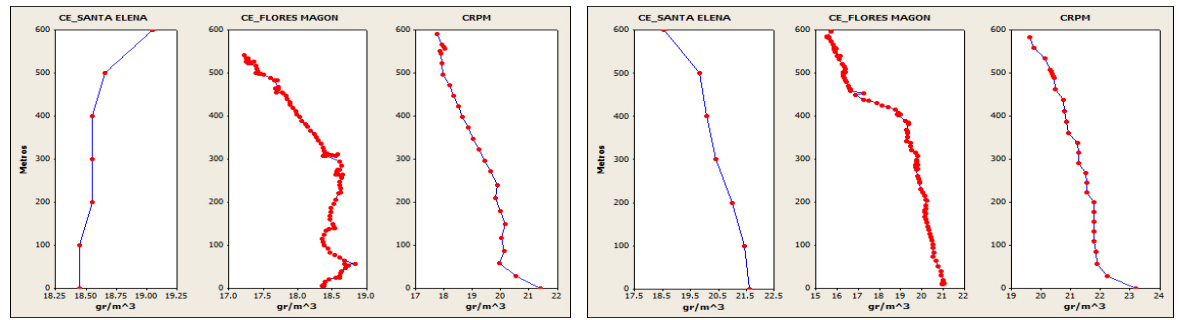

Figura 8. Perfiles de humedad absoluta a) 07 HL y b) 19 HL 29 de junio del 2015.

\section{COMENTARIOS FINALES}

La zona de estudio, además de ser costera, tropical y montañosa, presenta uno de los gradientes altitudinales más intensos del planeta, por lo que reviste interés y complejidad. Aquí se han presentado muestras de los efectos del relieve en las tendencias decadales de índices de precipitación pluvial y de temperatura. Si bien esas tendencias son congruentes con patrones mundiales y, en particular, con trabajos previos para una región más amplia que comprende a esta zona de estudio (Torres-Alavez et al., 2010; Vázquez-Aguirre et al., 2008), el relieve hace la diferencia: a media montaña y en el altiplano tanto la precipitación como el incremento térmico tienden a ser más intensos. Queda la interrogante de hasta qué punto esas tendencias gruesas se deben al calentamiento global, la variabilidad climática natural o los cambios en el uso del suelo.

Ante la incursión de un frente frío, se presenta disminución de la temperatura en la costa y en la montaña de manera que se acercan entre sí las curvas de la marcha diaria de las temperaturas, y se observa un arrastre de humedad hacia la zona montañosa. Dos días anteriores al frente la dirección del viento presenta un desfasamiento: en la costa se presenta viento del norte y en la montaña aun persiste la componente sur. Este desfasamiento desaparece el día del frente frío, cuando en ambos sitios se prevalece viento del norte. En el verano, ante el influjo de los alisios no perturbados, los procesos convectivos responden a la orografía. Durante la campaña de mediciones del 28 de junio al 3 de julio del 2015 se pudo observar cómo la nubosidad comienza en la cima de la zona montañosa y ahí mismo parece precipitar ( 2 de julio) o en algunos casos se traslada a la región costera ( 29 y 30 de junio), lo que no se mostró aquí por falta de espacio. Es común que surja un núcleo de nubosidad durante la mañana, ya sea sobre el mar (29 de junio) o sobre la costa ( 28 junio y 2 julio), que va desintegrándose mientras se mueve hacia el norte.

Las situaciones de estabilidad atmosférica en la parte baja de la atmósfera costera, quedan determinadas por el calentamiento (enfriamiento) diurno (nocturno), pero se espera que en la montaña o en el altiplano el relieve y su interacción con los alisios y 
brisas (marina y terral) decidirán las condiciones de convección o subsidencia, como lo documentaron Tejeda et al. (2004).

\section{AGRADECIMIENTOS}

Al proyecto Interacción superficielatmósfera en la zona montañosa central de la vertiente del Golfo de México: observaciones y modelación a alta resolución (CONACyT CB183040). Apoyaron en la instrumentación meteorológica y manejo de datos I. R. Méndez Pérez, G. Vázquez Cruz, I. M. García Martínez, B.Y. Mendoza Aguilar, M. Cortés Aguilar, A. Álvarez Pérez, A. Baruch Vera y M. Tejeda Zacarías.

\section{REFERENCIAS}

Alan, E. \& Martínez, M. (2010). Vegetación y uso de suelo. Universidad Veracruzana: http://cdigital.uv.mx/bitstream/123456789/9654/1/08VEGETACION.pdf

Martín-Vide, J. (2003). El tiempo y el clima. Barcelona: Rubes Editorial.

Mosiño, A. \& García, E. (1966). Evaluación de la sequía intraestival de la República Mexicana. Conferencia Regional Latinoamérica. Ciudad de México: Unión Geográfica Internacional.

Ruiz, A. Tejeda, A. Miranda, S. \& Flores, R. (2010). Climatología en Atlas del patrimonio natural, histórico y cultural de Veracruz. Eds. Florescano y J. Ortiz -Escamilla (editores). Xalapa: Gobierno del Estado de Veracruz.

Tejeda, A. Acevedo, F. \& Jáuregui, E. (1989). Atlas climático del estado de Veracruz. Xalapa: Universidad Veracruzana.

Tejeda, A. Méndez, I. Utrera, A. García, L. \& Mendoza, V. (2004). Circulación local y ascenso de aire en un sitio costero y tropical al pie de la montaña. En El clima entre el mar y la montaña. Santander, España: Asociación Española de Climatología.

Torres, J. Tejeda, A. Vázquez, J. Brunet, M. Hernández, A \& Ruíz, A. (2010). Índices de cambio climático y análisis de variabilidad en el estado de Veracruz, México. En Ciudad, clima y ecosistemas. Madrid, España: Asociación Española de Climatología.

Vázquez, J. Brunet, M. \& Jones. P. (2008). Cambios observados en los extremos climáticos de temperatura y precipitación en el estado de Veracruz, México a partir de datos diarios. En Cambio climático regional y sus impactos. Tarragona, España: Asociación Española de Climatología.

Williams, G. (2012). El bosque de niebla del centro de Veracruz: ecología, historia $y$ destino en tiempos de fragmentación y cambio climático. Xalapa, México: Instituto de Ecología A.C. 